\title{
Phase II study of ERC1671 plus bevacizumab versus bevacizumab plus placebo in recurrent glioblastoma: interim results and correlations with $\mathrm{CD} 4^{+}$T-lymphocyte counts
}

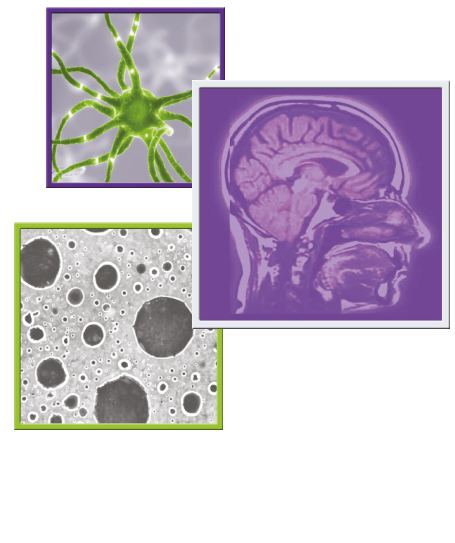

\author{
Daniela A Bota*,1,2,3, Jinah Chung ${ }^{3}$, Manisha Dandekar ${ }^{3}$, Jose A Carrillo1,2,3, Xiao-Tang \\ Kong ${ }^{1,2,3}$, Beverly D Fu ${ }^{1,3}$, Frank PK Hsu ${ }^{2,3}$, Axel H Schönthal ${ }^{4}$, Florence M Hofman ${ }^{5}$, \\ Thomas C Chen ${ }^{6}$, Raphael Zidovetzki ${ }^{7}$, Chrystel Pretto ${ }^{8}$, Ankie Strik ${ }^{6,8}$, Virgil EJC Schijns ${ }^{8,9}$ \\ \& Apostolos Stathopoulos $8,9,10$ \\ ${ }^{1}$ Department of Neurology, University of California Irvine, Irvine, CA 92868, USA \\ ${ }^{2}$ Department of Neurological Surgery, University of California Irvine, Irvine, CA 92868, USA \\ ${ }^{3}$ Chao Family Comprehensive Cancer Center, University of California Irvine, Irvine, CA 92868, USA \\ ${ }^{4}$ Department of Molecular Microbiology \& Immunology, Keck School of Medicine, University of Southern California, Los Angeles, \\ CA 90033, USA \\ ${ }^{5}$ Department of Pathology, Keck School of Medicine, University of Southern California, Los Angeles, CA 90033, USA \\ ${ }^{6}$ Department of Neurosurgery, Keck School of Medicine, University of Southern California, Los Angeles, CA 90033, USA \\ ${ }^{7}$ Cell Biology \& Neuroscience, University of California, Riverside, CA 92507, USA \\ ${ }^{8}$ Epitopoietic Research Corporation, Gembloux, 5032 Isnes, Belgium \\ ${ }^{9}$ Cell Biology \& Immunology Group, Wageningen University, 6708 Wageningen, The Netherlands \\ ${ }^{10}$ Department of Neurosurgery, Euroclinics Hospital, 15121 Athens, Greece \\ *Author for correspondence: Tel.: +1 714456 7032; Fax: +1 714456 6894; dbota@uci.edu
}

\begin{abstract}
Aim: ERC1671 is an allogeneic/autologous therapeutic glioblastoma (GBM) vaccine - composed of whole, inactivated tumor cells mixed with tumor cell lysates derived from the patient and three GBM donors. Methods: In this double-blinded, randomized, Phase II study bevacizumab-naive patients with recurrent GBM were randomized to receive either ERC1671 in combination with granulocyte-macrophage colonystimulating factor (GM-CSF) (Leukine ${ }^{\circledR}$ or sargramostim) and cyclophosphamide plus bevacizumab, or placebo plus bevacizumab. Interim results: Median overall survival (OS) of patients treated with ERC1671 plus bevacizumab was 12 months. In the placebo plus bevacizumab group, median OS was 7.5 months. The maximal CD4 ${ }^{+}$T-lymphocyte count correlated with OS in the ERC1671 but not in the placebo group. Conclusion: The addition of ERC1671/GM-CSF/cyclophosphamide to bevacizumab resulted in a clinically meaningful survival benefit with minimal additional toxicity.
\end{abstract}

First draft submitted: 19 June 2018; Accepted for publication: 17 July 2018; Published online: 29 August 2018

Keywords: allogeneic • autologous • bevacizumab • CD4+ T lymphocyte • ERC1671 • GBM • GBM vaccine • glioma surgery $\bullet$ immunotherapy

Glioblastoma (GBM, WHO grade IV astrocytic glioma) is the most common and most aggressive form of brain cancer in adults. The annual incidence is about 3.19 cases per 100,000 population, resulting in approximately 10,000 new cases each year in the USA [1]. Prognosis for GBM is very poor. For decades, the mainstay of therapeutic intervention was based on surgical resection (when safely feasible), followed by radiotherapy (RT). In 2005, data from the landmark European Organisation for Research and Treatment of Cancer - National Cancer Institute of Canada (EORTC-NCIC) trial changed the standard of care treatment for GBM. This Phase III trial demonstrated a survival advantage for concomitant and adjuvant temozolomide (TMZ) chemotherapy when added to the standard course of radiation [2]. In the group of patients assigned to radiation plus TMZ, median survival improved from 12.1 (RT alone) to 14.6 months. 2-year and 5-year survival was 27 and $9.8 \%$, respectively, as compared with 10.9 and $1.9 \%$ for radiation alone $[2,3]$. The latest therapy to be approved for GBM in the USA and Europe is the

Future Medicine 
alternating electric fields generator NovoTTF/Optune ${ }^{\circledR}[4]$, which may extend median overall survival (OS) by about 5-24 months [5].

However, despite all advancements in GBM care, the vast majority of patients relapse. At the time of recurrence after the first-line therapy, further treatment options are limited [6,7]. Repeat surgery is often considered, but tumor cells infiltrating the brain and spinal cord many times prevent a significant surgical resection. At the same time, invasive tumor cells appear to be more resistant to cytotoxic drug therapy and to have a higher proliferative potential. In general, the treatment of recurrent GBM by repeat surgery, re-irradiation and further chemotherapy may increase the symptom-free interval and moderately extend OS, primarily in patients with good performance status $[8,9]$.

The only US FDA targeted treatment approved for recurrent GBM patients is the angiogenesis inhibitor bevacizumab, a humanized monoclonal antibody targeting VEGF [10]. When used alone or in combination with a cytotoxic agent, it improves imaging parameters for most patients, but duration of benefits is transient and short lived. Its impact on prolonging OS appears limited, especially when used outside the clinical trial settings [1113]. Although, bevacizumab is approved for recurrent GBM in the USA and Canada, it did not receive market authorization by the EMA.

In recent years, immunotherapy of cancers has garnered much increased attention as a new pillar of cancer treatment, with the potential to assume a place alongside surgery, RT and chemotherapy [14,15]. We have been developing ERC1671 (Gliovac ${ }^{\mathrm{TM}}$ ) as a novel approach to GBM therapy. ERC1671 is a course of vaccines, where irradiated/inactivated tumor cells are combined with tumor cell lysate for subcutaneous injection. The tumor cells and lysates are derived from the GBM patient to be treated (autologous component), as well as from three other GBM patient donors (allogeneic component). This mix is administered together with cyclophosphamide and granulocyte-macrophage colony-stimulating factor (GM-CSF) to support immune system priming.

Preclinical proof of principle of the concept underlying the ERC1671 vaccination approach was established 10 years ago in rat models, where it was shown that allogeneic GBMs can be used to vaccinate against an established syngeneic tumor [16]. The first published report [17] on ERC1671's use in the clinical setting described the vaccine's effect in a recurrent GBM patient who previously had failed second-line bevacizumab. Although similar patients generally are moribund within a few short weeks within bevacizumab failure, the ERC1671-treated patient survived for 10 months without any other adjuvant therapy, but eventually died of complications related to his previous chemotherapies [17]. In a related study [18], nine recurrent GBM patients were treated with ERC1671 on a compassionate use exemption protocol. The majority of these patients was from European countries and therefore did not receive bevacizumab either before or during the course of vaccination. 6-month survival on the ERC1671 regimen was $100 \%$, and 12 -month survival was $40 \%$, providing initial evidence of low toxicity and promising activity of this new therapeutic approach [18].

To further investigate and validate safety and effectiveness of ERC1671, a Phase II, double-blinded, placebocontrolled clinical study (NCT01903330) was initiated at the University of California, Irvine. Because in the USA, bevacizumab is an approved treatment in the recurrent setting, bevacizumab was included, and so was cyclophosphamide and GM-CSF to support immune system priming. In this report, we are presenting interim results from this trial, based on nine patients who were unblinded as stipulated by the study protocol at the time of further progression.

\section{Methods}

Composition of vaccine

ERC1671 (Gliovac) is an immunological therapy composed of primary irradiated/inactivated whole tumor cells and lysates from allogeneic and autologous GBM patients, administered in combination with the immune system priming agents cyclophosphamide and GM-CSF. Specifically, the complete ERC1671 regimen consists of: inactivated tumor cells and tumor cell lysate from the patient to be treated (autologous component: ERC-D); inactivated tumor cells and tumor cell lysate from three other GBM patients (allogeneic components: ERC-A from donor X, ERC-B from donor $\mathrm{Y}$ and ERC-C from donor Z); cyclophosphamide to relax the immune-suppressive environment; and GM-CSF to enhance immune responses.

The ERC1671 vaccine is administered by intradermal vaccination. One dose of ERC1671 (i.e., ERC-A through D) consists of whole tumor cells (between $1 \times 10^{5}$ and $1 \times 10^{6}$ cells) combined with tumor cell lysate (between $1 \times 10^{5}$ and $1 \times 10^{6}$ cells). Immediately prior to injection, $500 \mu \mathrm{g} \mathrm{GM-CSF}$ (Leukine ${ }^{\circledR}$ ) is added to each vaccine dose, and the combined volume is injected together. Cyclophosphamide (Cytoxan $\left.{ }^{\circledR}\right)$ is given orally $(2 \times 25 \mathrm{mg}$ 


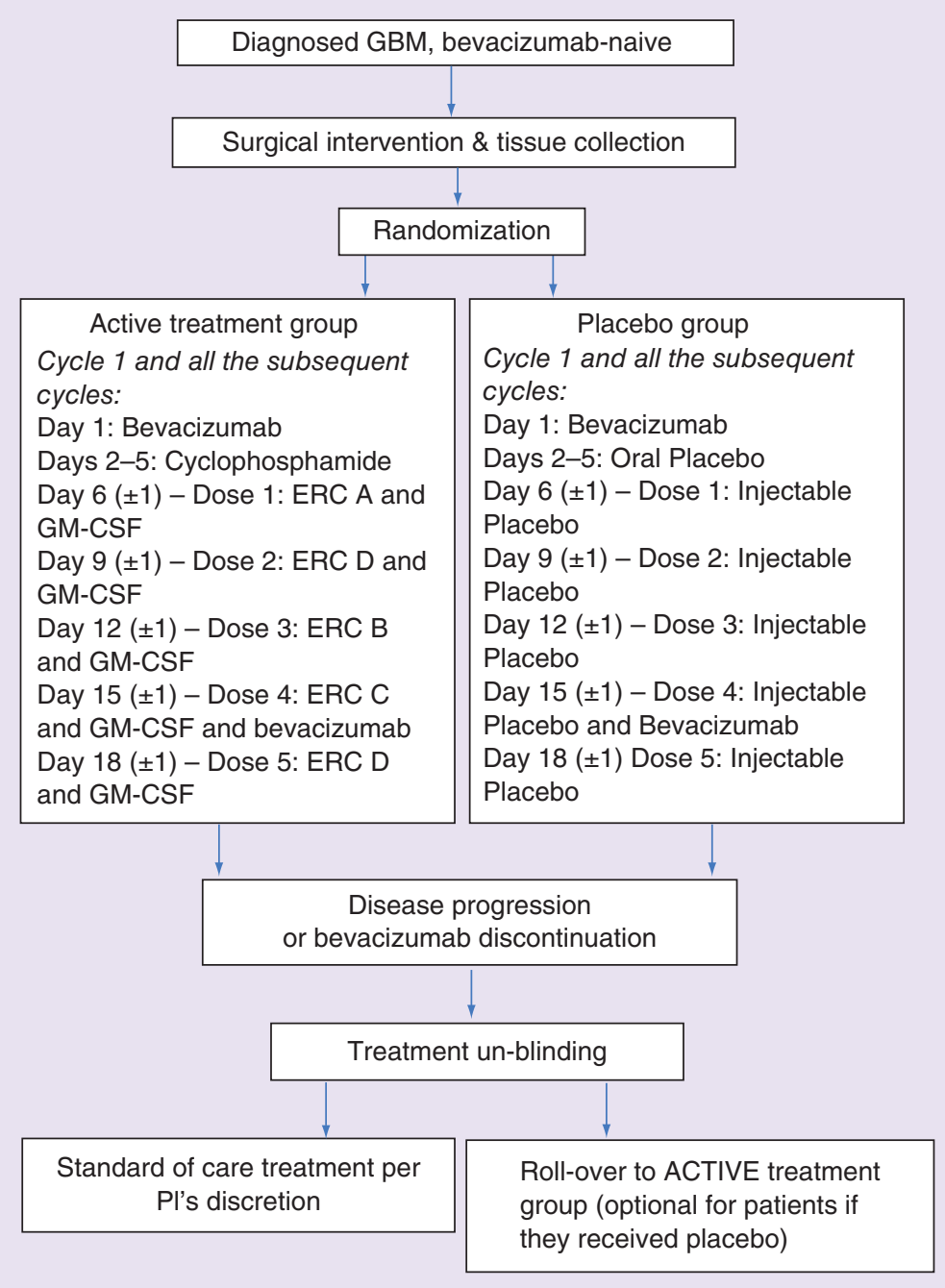

Figure 1. Study schema. Each treatment cycle is 28 days long. The first day of the first cycle is scheduled 29 days from surgery, which satisfies the US FDA mandated waiting time of 4 weeks between surgery and first use of bevacizumab, and allows for sufficient time to process the patient's tumor tissue for vaccine production. The timeline for days 1 through 5 (bevacizumab, followed by cyclophosphamide) is implemented strictly, whereas subsequent administration of each individual dose of vaccine (in combination with GM-CSF) is flexible by \pm 1 day. ERC-D is the autologous component, whereas ERC-A, $-B$ and $-C$ are allogeneic components from three different GBM patient donors. Both groups, active treatment group and placebo group, receive bevacizumab on day 1 and $15( \pm 1)$ of each cycle. This course of treatment is repeated every 28 days until disease progression or intolerance, at which time assignment of the respective patient will be unblinded.

GBM: Glioblastoma; GM-CSF: Granulocyte-macrophage colony-stimulating factor.

Cytoxan capsules per day) for 4 days (days 2-5) at the beginning of each cycle (see treatment scheme Figure 1). For the control patient group, all doses ERC-A through ERC-D are replaced by placebo treatment, which contains injectable freezing medium only, supplemented with sucrose and human albumin. No GM-CSF is added. Oral cyclophosphamide is replaced by oral placebo. Patients in both groups, active treatment group as well as placebo group, receive $10 \mathrm{mg} / \mathrm{kg}$ bevacizumab (Avastin ${ }^{\circledR}$ ) infusion on day 1 and 15 of each 28 -day cycle. Treatment cycles are continued until progression of disease or intolerance, at which time group assignments are unblinded.

\section{Vaccine production}

ERC1671/Gliovac is being manufactured under good manufacturing practice (GMP) approved aseptic conditions from surgically removed GBM tissues. These tissues are received and released by a tissue bank of human body mate- 
rial, after testing for absence of transmissible infections, including HIV, HBV, HCV, CMV, HTLV and Treponema pallidum/syphilis. After coding by a suitable anonymization procedure, samples are sent under temperaturecontrolled conditions to the GMP manufacturing site. Cells are isolated by mechanical dissection and washed in Earl's balanced salt solution. Isolated cells are counted and haptenized with 1-fluoro 2,4-dinitrofluorobenzene to improve immunogenicity. Haptenized cells are divided into two equal parts. One part is preserved for freezing in a sucrose medium, and the other part is lysed by osmotic shock. Both parts are irradiated with 25 gray of gamma radiation to inactivate any replication competence. Thereafter, all preparations are stored at $-80^{\circ} \mathrm{C}$.

\section{Patient characteristics \& selection}

For patients to be eligible for inclusion in the trial, they must have histologically confirmed WHO grade IV malignant glioma and documented treatment failure to standard of care treatment, including surgery followed by RT with concomitant and adjuvant TMZ chemotherapy. As well, these relapsed GBM patients must be bevacizumabnaive, aged $\geq 18$ years, have measurable contrast-enhancing tumor on MRI and Karnofsky Performance Status of $\geq 70 \%$. Patients must have normal organ and marrow function as defined by hemoglobin $>9.0 \mathrm{~g} / \mathrm{dl}$, leukocytes $>1,500 / \mathrm{mcl}$, absolute neutrophil count $>1,000 / \mathrm{mcl}$, platelets $>125,000 / \mathrm{mcl}$; total bilirubin within normal institutional limits, serum creatinine $>1.5 \mathrm{mg} / \mathrm{dl}$ and aspartate aminotransferase (AST or SGOT) $/$ serum glutamic pyruvic transaminase (SGPT or ALT) $<2.5 \times$ institutional upper limit of normal. Systemic corticosteroid therapy must be at a dose of $\leq 4 \mathrm{mg}$ of dexamethasone or equivalent per day during the week prior to the first day of initiation of the first vaccination cycle.

\section{Procedures}

All patients described in the current report were enrolled and treated at the University of California Irvine, CA, USA, under an institutional review board approved protocol and after signing appropriate institutional review board approved informed consent forms.

Each treatment cycle is 28 days long. The first day of the first cycle (which starts the cycle by infusion of bevacizumab) is scheduled 29 days after the surgery. This satisfies the FDA-mandated waiting time of 4 weeks between surgery and first use of bevacizumab. In addition, it provides sufficient time to process the tumor tissue for vaccine production and ensures the availability of the ERC-D (autologous) component of the treatment regimen (which is administered for the first time on day 9 of the first cycle). The three allogeneic vaccine components (ERC-A, - B, -C) are in stock and readily available when needed. The treatment is repeated every 28 days until progression of disease or intolerance. Humoral immunologic response is measured at baseline and at selected times following vaccination and at the time of disease progression or end of treatment (EOT). Patients undergo brain MRI as part of standard care before starting cycle 1 and every 8 weeks until disease progression, and whenever progression is suspected based on clinical symptoms. Tumor response is assessed using both the Macdonald and the iRANO response criteria for high-grade gliomas $[19,20]$, which considers radiologic imaging, neurological status and steroid dosing. Safety is evaluated throughout the trial by the incidence of adverse events (AEs), physical examination findings, vital signs and clinical laboratory test results. AEs are graded for severity using NCI Common Terminology Criteria for Adverse Events v.4.0 [21].

At the time of proven disease progression, the group assignment of these patients is unblinded as per protocol stipulation. At this time, patients from the active treatment group are offered standard or palliative care, whereas patients from the placebo group are offered the opportunity to roll over to the active treatment group. Patients who do not participate in the rollover option of the study return for an end-of-therapy visit where alternate treatment and/or care options are discussed. Patients continue to be followed for survival.

\section{Outcomes}

OS is measured from day 1 of Cycle 1 until death. Progression-free survival (PFS) is defined as the time from day 1 of Cycle 1 to the date of progression or death due to any cause. Immune monitoring in the peripheral blood (including, but not limited to $\mathrm{CD}^{+}{ }^{+} \mathrm{T}$ lymphocyte counts) was performed every 2 weeks.

\section{Statistical analysis}

The survival data were plotted as Kaplan-Meier survival curves and analyzed for significance using logrank test. 
Table 1. Patient characteristics.

\begin{tabular}{|c|c|c|}
\hline Characteristics & Active treatment group + bevacizumab $(n=5)$ & Placebo control + bevacizumab $(n=4)$ \\
\hline Age (average [range]) & $59(49-65)$ & $57(48-74)$ \\
\hline Male (n [\%]) & $4(80 \%)$ & $3(75 \%)$ \\
\hline KPS (average [range]) & $80(70-100)$ & $90(70-100)$ \\
\hline \multicolumn{3}{|l|}{ Relapses (n [\%]) } \\
\hline 1 & $5(100 \%)$ & $3(75 \%)$ \\
\hline IDH1/2 status (wild-type [\%]) & $5(100 \%)$ & $4(100 \%)$ \\
\hline MGMT promoter & $\begin{array}{l}\text { Unmethylated } 4(80 \%) \\
\text { Undetermined } 1(10 \%)\end{array}$ & $\begin{array}{l}\text { Unmethylated } 3(75 \%) \\
\text { Undetermined } 1(25 \%)\end{array}$ \\
\hline
\end{tabular}

\section{Results}

Study design

Presented here are interim results (as per 1 September 2017) of an ongoing, Phase II, double-blinded, placebocontrolled study of a novel cancer therapeutic vaccine, ERC1671/Gliovac, in patients with recurrent, bevacizumabnaive GBM. In the active treatment group, vaccination is combined with GM-CSF, cyclophosphamide and bevacizumab, whereas the control group receives placebo and bevacizumab only (Figure 1).

The key principle underlying this particular vaccination approach is the use of a broad set of tumor antigens, derived from freshly resected whole tumor tissue - not only from the patient under treatment, but expanded to include the same from three independent GBM tissue donors. This multivalent array of autologous and allogeneic antigens is expected to reduce the chance of immune escape, which can emerge from antigenic loss or active major histocompatibility complex (MHC) downregulation and is more likely to occur when using a single- or limitedantigen targeted immunotherapy. During each immunization cycle, the immune effector response is triggered by breaking tolerance to the patient's tumor antigens through first injecting one of the allogeneic components (i.e., ERC-A). This is facilitated by the fact that the injection of allogeneic preparation evokes a strong anti-nonself immune response. Thereafter, the second vaccine dose is patient-derived (autologous ERC-D) to focus the triggered immune reaction toward the patient's tumor antigens. This is followed by two additional (booster) injections of allogeneic material (ERC-B and -C) and a final injection of ERC-D (Figure 1).

For each cycle, the immunizations are preceded by a short regimen of low-dose, metronomic cyclophosphamide, based on the published literature which shows that low-dose cyclophosphamide stimulates dendritic cell expansion, contributes to the induction of antitumor cytotoxic $\mathrm{T}$ lymphocytes and depletes immune-inhibitory immune cells and stimulates the polarization of $\mathrm{CD}^{+} \mathrm{T}$ cells into $\mathrm{TH} 1$ and/or TH17 lymphocytes eventually affecting the Treg/T-effector ratio in favor of tumor regression [22,23]. In addition, each dose of tumor antigens is accompanied by co-injection of GM-CSF. This growth factor has been shown to effectively and potently enhance the immune response in several different systems [24,25]. The rationale for also including bevacizumab is based on earlier observations that VEGF inhibits immune activity via inhibitory actions upon dendritic cells [26]. As such, using anti-VEGF therapy via administration of bevacizumab to cancer patients can enhance dendritic cell and T-cell responses to antigens [27], and potentially increase the level and specificity of the immunostimulation achieved by ERC1671.

As per treatment protocol, nine study participants were recently unblinded. Characteristics of these patients are summarized in Table 1. Unblinding revealed that four patients had received ERC1671 vaccine, four had received placebo. One patient was marked as nonevaluable due to discontinuation prior to completion of the first cycle.

\section{Clinical safety}

Clinical results for toxicity show an equal distribution of AEs between the active treatment and placebo groups, with no grade 4 or 5 toxicities (Table 2). Among documented grade 3 toxicities, headaches were the most common. Among all toxicities, injection site reactions (induration, erythema and ulceration) were most frequently noted. Although these skin reactions were mild, they indicated the development of immune responses. However, they were not consistently noted in all patients, and hence no clear correlation between efficacy and erythema response can be concluded. Similarly, other observed mild systemic reactions, including self-limiting fever and chills, represent expected outcomes related to the intended immune stimulation. 


\begin{tabular}{|c|c|c|}
\hline Adverse events (ERC1671 plus bevacizumab) & Grade 3 & Total \\
\hline Injection site reaction & 0 & 67 \\
\hline Arthralgia & 0 & 70 \\
\hline Gait disturbance/fall & 1 & 4 \\
\hline Back pain & 1 & 6 \\
\hline Headache & 2 & 9 \\
\hline Anxiety & 0 & 6 \\
\hline Total events of any grade & 4 & 162 \\
\hline Adverse events (placebo plus bevacizumab) & Grade 3 & Total \\
\hline Gait disturbance/fall & 1 & 38 \\
\hline Muscle weakness & 2 & 4 \\
\hline Hydrocephalus & 2 & 7 \\
\hline Delirium & 1 & 1 \\
\hline Urinary incontinence & 1 & 4 \\
\hline Thromboembolic event & 1 & 4 \\
\hline Total events of any grade & 8 & 58 \\
\hline
\end{tabular}

\section{Clinical efficacy: radiology data}

The patients were monitored with imaging every 8 weeks $(2$ cycles) as stated by protocol. The overall response rate was higher for the ERC 1671 arm versus the control arm at 75\% (3/4) versus 25\% (1/4). The responders in the ERC 1671 group experienced durable responses - as exemplified in Figure 2. The patient in this case has achieved a partial response after cycle 1, and has maintained his response for more than 7 months. The patient's OS is now over 2 years.

\section{Clinical efficacy: OS}

Median OS of patients treated with ERC1671 plus bevacizumab was 12.1 months, with one patient surviving $>2$ years. In the group treated with placebo plus bevacizumab, median OS was shorter at 7.6 months, with all patients having succumbed within 1 year (Figure 3). Median PFS of the treated with ERC1671 + bevacizumab was 7.3 months (223 days), compared with the patients treated with placebo + bevacizumab, where median PFS was 5.4 months (164 days) (data not shown).

\section{Clinical efficacy: immune correlations}

$\mathrm{CD}^{+} / \mathrm{CD}^{+}$helper T-lymphocytes counts were monitored in peripheral blood at the baseline and every 2 weeks during the study participation. The maximum count (cells $/ \mu \mathrm{l})$ is defined as the highest value measured in a patient during the study participation, while the EOT count is the $\mathrm{CD} 3 / \mathrm{CD}^{+}$helper T-lymphocytes count $(\mathrm{cells} / \mu \mathrm{l})$ at the EOT visit. Both the maximum count and the EOT count highly correlated with the OS in the patients treated with ERC1671 and bevacizumab, but not in the placebo and bevacizumab group (see Figure 4).

\section{Discussion}

The present study provides preliminary evidence that ERC1671/gliovac immunotherapy, combined with bevacizumab, is safe and potentially effective in recurrent GBM patients. A number of bevacizumab-based clinical trials were recently completed, all of them with a medium OS at 12 months between 10 and 26\% [28-30], very similar with our control arm results (only one out of four patients survived 12 months). In comparison, our active treatment group patients had a 12 months OS of 50\%, very similar with our previously published Phase 0 data [18].

Our study also suggests that the response to the ERC1671 vaccine treatment directly correlates with the $\mathrm{CD}_{4}^{+}$ helper T-lymphocytes counts in the peripheral blood. The role of $\mathrm{CD}^{+}{ }^{+} \mathrm{T}$-cell response in antitumor immunity is well-described in the literature [31]. In animal models, the presence of $\mathrm{CD}^{+}$helper $\mathrm{T}$ lymphocytes is essential for eliciting a response to cell-based vaccines [32,33]. In GBM, $\mathrm{CD}^{+}$tumor infiltration after vaccination was reported for HSPPC-96 dendritic cell vaccine [34]. Also, a recent preclinical study has shown that, while $\mathrm{CD} 4^{+}, \mathrm{CD}^{+} \mathrm{T}$ and NK-cell subsets are required early to establish anti-GBM immune responses, only $\mathrm{CD}^{+}{ }^{+} \mathrm{T}$ cells are continuously 


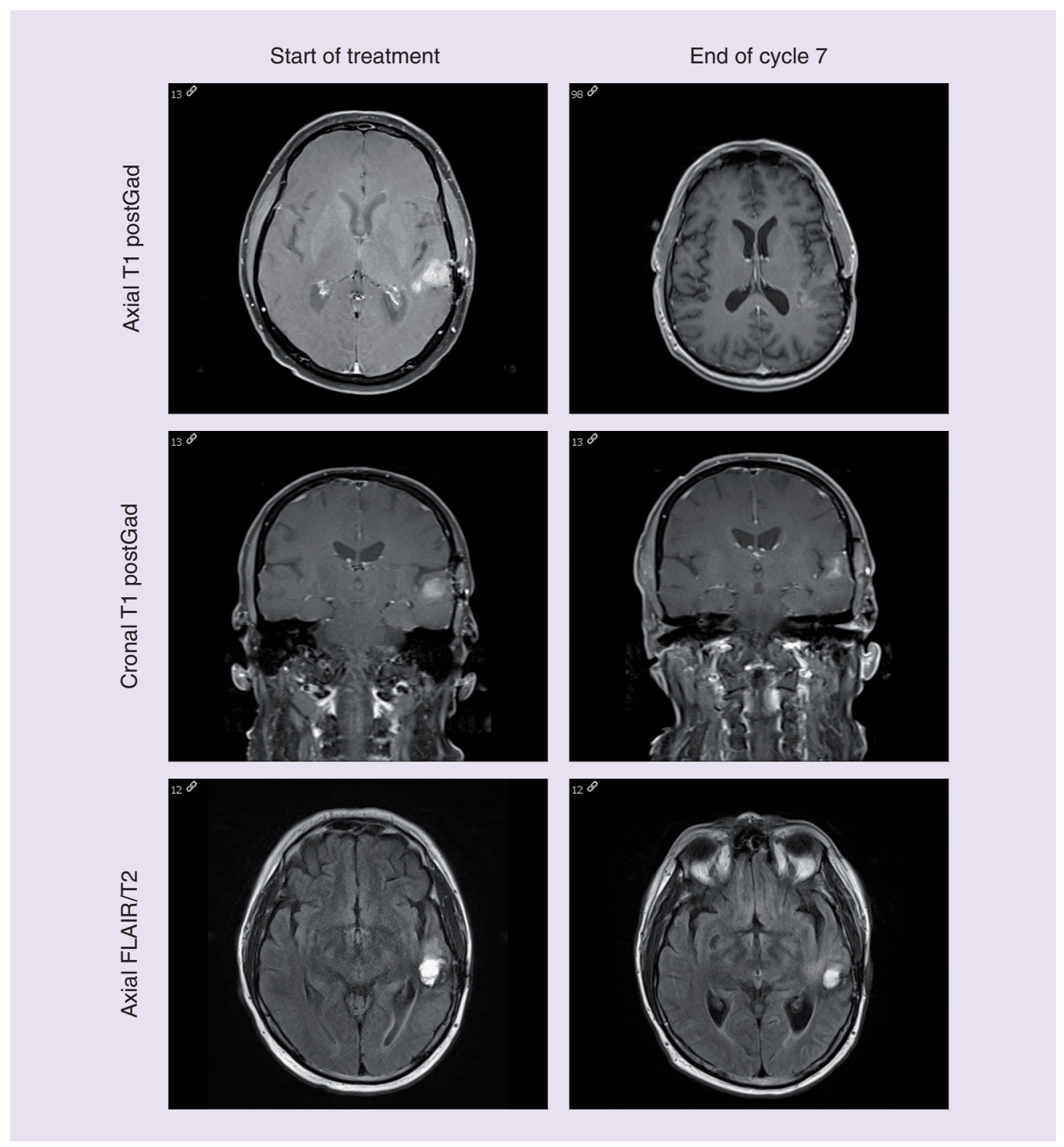

Figure 2. MRI of the brain for a patient randomized to the ERC1671/bevacizumab arm. Showing the tumor size before starting in the ERC1671/bevacizumab treatment and end of cycle 7. The MRI shows significant decrease in contrast enhancement over time and stable fluid-attenuated inversion recovery signal.

required to achieve maximal survival in this immunocompetent GBM model treated with radiation and PD-1 blockade [35]. We have also reported T-lymphocyte infiltration in our first ERC1671 publication (single-patient report) [17]. However, the importance of monitoring $\mathrm{CD}^{+}$helper $\mathrm{T}$ lymphocytes in the peripheral blood is not well established in GBM clinical trials of cell-based vaccines, and might represent a biomarker of response for our therapeutic strategy.

The rationale underlying the ERC1671 vaccine is to evoke an oligoclonal and partly allo-specific immune induction, based on the use of a broad set of tumor antigens derived from freshly resected GBM tumor tissues from patient and three unrelated donors. This broad antigen-based approach differs from many other currently ongoing attempts at developing a tumor vaccine, some of which zero in on one or only a few individual, more or less tumor-specific targets, such as SurVaxM (aimed at surviving) or rindopepimut, a peptide vaccine aimed at the EGFR deletion mutation EGFRvIII [36,37].

In developing ERC1671, we preferred a multimodular approach that is based on syngeneic lysates and cells, mixed with lysates and cells from three different allogeneic tumor donors. This mixture of antigens is not defined, but expected to overlap to a large degree with the specific tumor antigens in the patient. Moreover, this strategy enables triggering of an immune response against a broad array of tumor antigens and also triggers nonself 
(A)
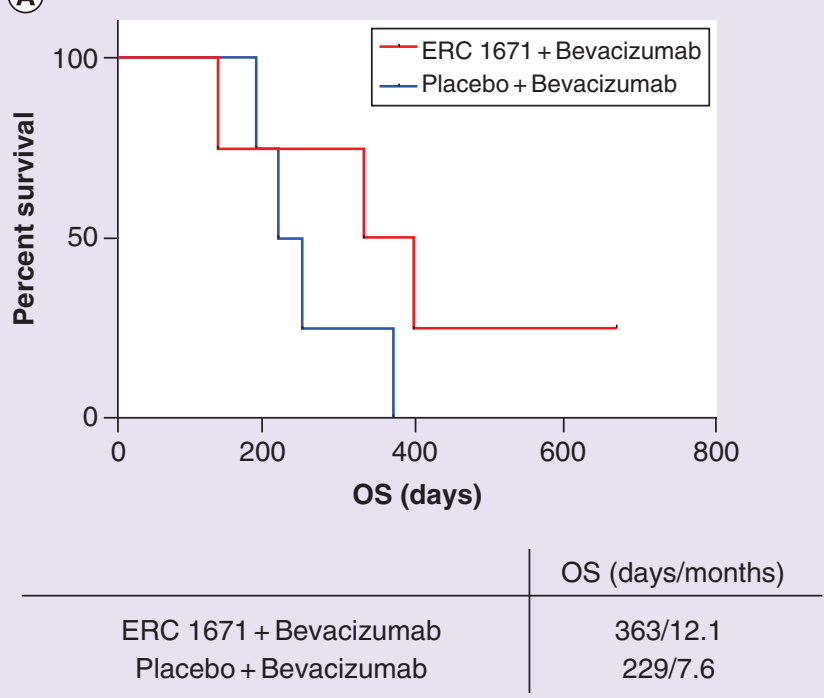

(B)

\begin{tabular}{l|l} 
Treatment & OS12 \\
\hline Bevacizumab only studies & $10-26 \%$ \\
\hline Taal (BELOB) [28] & $26 \%$ \\
Field (CABARET) [29] & $24 \%$ \\
Heiland (Germany) [30] & $10 \%$ \\
\hline ERC1671 compassionate use [18] & $40 \%$ \\
ERC1671 plus Bevacizumab & $50 \%$
\end{tabular}

Figure 3. Overall survival and comparisons with previous studies. (A) Median OS was 363 days in the active treatment group (4 patients), compared with 229 days in the placebo group (4 patients). (B) A total of 12 months OS in the ERC1671/bevacizumab arm of the study is superior to previously published single-arm bevacizumab studies.

OS: Overall survival.

triggered allo-immune reactivity, a classical allograft-directed immune response, typical for nonmatching major histocompatibility between graft cells and the host. Cyclophosphamide is used in this protocol because this drug was shown to diminish the Treg population, and thereby decrease the immune suppressive environment of the tumor [22]. GM-CSF has been used to effectively and potently enhance the immune response in several different systems and therefore will be administered as part of this protocol [38].

In summary, preliminary analysis of interim results from our study indicates that the addition of ERC1671/GM$\mathrm{CSF} /$ cyclophosphamide to bevacizumab resulted in a clinically meaningful survival benefit with minimal additional toxicity. The study is ongoing with the anticipated addition of two other sites.

\section{Future perspective}

The last years have revolutionized the field on cancer immunotherapies, with novel treatments being approved almost every month for a variety of malignancies, including melanoma, lung cancer, bladder cancer, etc. However, in spite of multiple Phase III studies, no GBM immunotherapy has been able to show effectiveness and to obtain FDA approval. Our approach brings a different concept - namely the use of a broad antigen-based approach, including both allogeneic and autologous components and shows promise in activating a very important population of cells - namely the $\mathrm{CD}^{+}$helper $\mathrm{T}$ lymphocytes. The future promise of our treatment might also rest in the ability to combine it with bevacizumab, and potentially with immune checkpoint inhibitors - an option that will allow more 
(A)
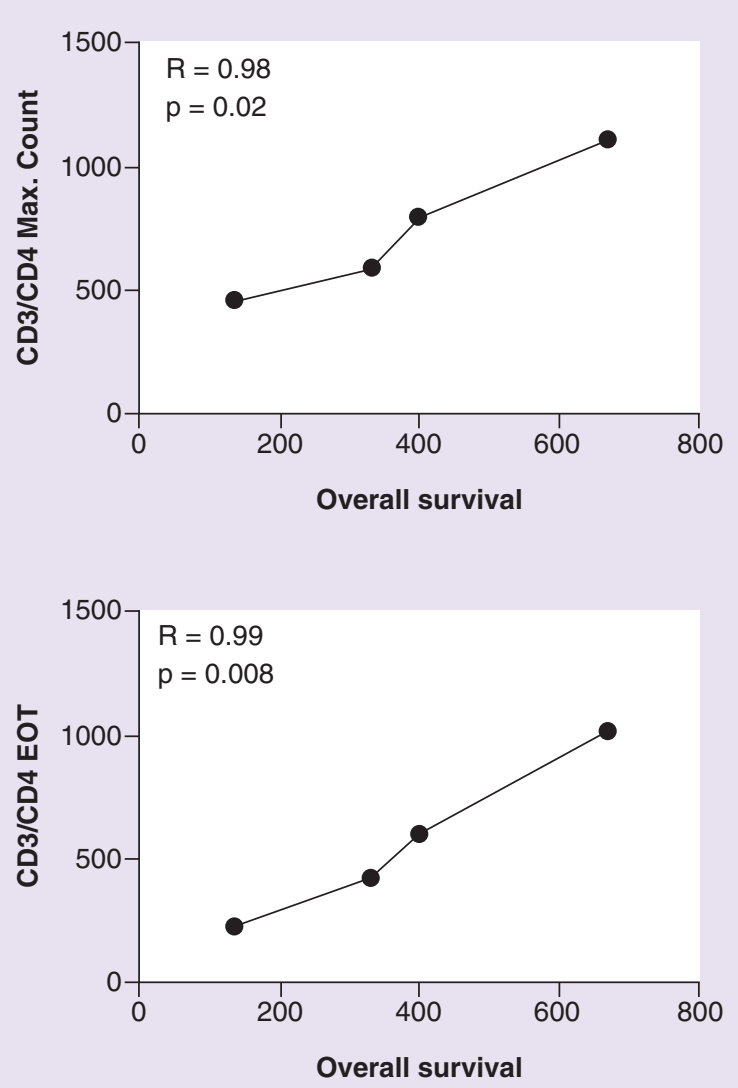

(B) Placebo plus Bevacizumab
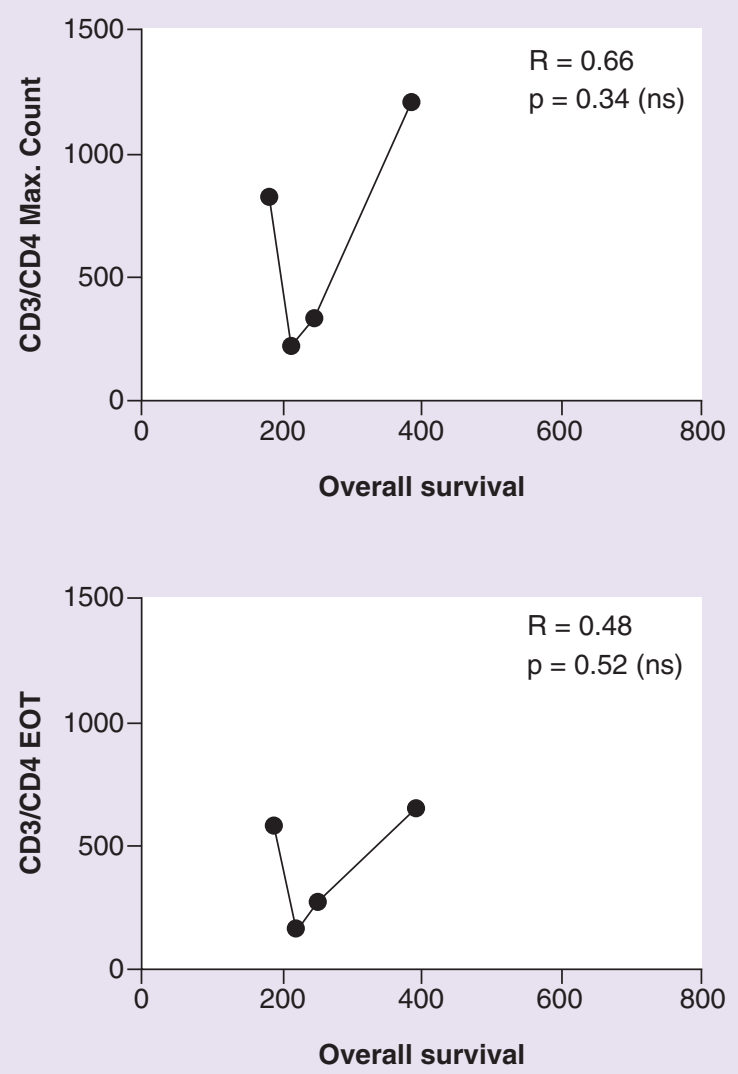

Figure 4. $\mathrm{CD}^{+} / \mathrm{CD} 4^{+}$lymphocyte counts highly correlate with overall survival in the ERC1671/bevacizumab treatment arm. The maximal values, as well as end-of-treatment values, of $\mathrm{CD}^{+} / \mathrm{CD}^{+}$mature helper/inducer $\mathrm{T}$ lymphocytes were determined in all patients from the ERC1671/bevacizumab group (A) and the placebo/bevacizumab group (B). Cell numbers were plotted over overall survival time.

powerful immune activation in the periphery as well as more aggressive local tumor immunological targeting and destruction.

\section{Summary points}

- Immunotherapies for glioblastoma (GBM) are currently being developed.

- ERC1671 is a novel immunotherapy concept, combining allogeneic/autologous components - whole, inactivated tumor cells mixed with tumor cell lysates derived from the patient and three GBM donors, in addition to immune priming with Granulocyte-macrophage colony-stimulating factor (GM-CSF) (Leukine ${ }^{\circledR}$, Sanofi Genzyme, MA, USA) and low-dose cyclophosphamide.

- This experimental immunotherapy is very well tolerated, with minimal side effects.

- In the active treatment group (ERC1671 plus bevacizumab), overall survival is correlated with the maximal CD4 ${ }^{+}$ T-lymphocyte count in the peripheral blood, highlighting the role of $\mathrm{CD}^{+}{ }^{+}$lymphocyte in achieving and maintaining immunologic tumor control.

- The combination of ERC1671 and bevacizumab had encouraging overall survival results, when compared with the bevacizumab alone group in our study and with recently published bevacizumab studies.

- Recurrent GBM patients might benefit from participation in personalized immunotherapy studies such as the one presented in this article. 
Financial \& competing interests disclosure

This study was supported by funding from Epithopoietic Research Corporation. The study is an investigator-initiated study (DA Bota) funded by Epitopoietic Research Corporation (ERC). DA Bota has designed the study in collaboration with the study sponsor and the other investigators and managed the clinical trial database, and performed statistical analysis (with R Zidovetzki support). The corresponding author had full access to all the data in the study and had final responsibility for the decision to submit for publication. The following authors are on the ERC advisory board, board of directors, employed by ERC or own ERC shares: TC Chen, C Pretto, V EJC Schijns and A Stathopoulos. Grant number: UCI Cancer Center Award [P30CA062203] from the National Cancer Institute. The authors have no other relevant affiliations or financial involvement with any organization or entity with a financial interest in or financial conflict with the subject matter or materials discussed in the manuscript apart from those disclosed. No writing assistance was utilized in the production of this manuscript.

\section{Acknowledgements}

The following authors have contributed significantly to the experimental design (DA Bota, V EJC Schijns and A Stathopoulos), its implementation (DA Bota, J Chung, M Dandekar, JA Carrillo, XT Kong, BD Fu, FPK Hsu), or analysis and interpretation of the data (all authors). All authors were involved in the writing of the manuscript at draft and any revision stages, and have read and approved the final version.

\section{References}

1. CBTRUS. Primary Brain and Central Nervous System Tumors Diagnosed in the United States in 2004-2007. Central Brain Tumor Registry of the United States, IL, US (2011).

2. Stupp R, Mason WP, van den Bent MJ et al. Radiotherapy plus concomitant and adjuvant temozolomide for glioblastoma. $N$. Engl. J. Med. 352, 987-996 (2005).

3. Stupp R, Hegi ME, Mason WP et al. Effects of radiotherapy with concomitant and adjuvant temozolomide versus radiotherapy alone on survival in glioblastoma in a randomised Phase III study: 5-year analysis of the EORTC-NCIC trial. Lancet Oncol. 10, 459-466 (2009).

4. Swanson KD, Lok E, Wong ET. An overview of alternating electric fields therapy (NovoTTF Therapy) for the treatment of malignant glioma. Curr. Neurol. Neurosci. Rep. 16, 8 (2016).

5. Stupp R, Taillibert S, Kanner AA et al. Maintenance therapy with tumor-treating fields plus temozolomide vs temozolomide alone for glioblastoma: a randomized clinical trial. JAMA 314, 2535-2543 (2015).

6. Gallego O. Nonsurgical treatment of recurrent glioblastoma. Curr. Oncol. 22, e273-281 (2015).

7. Weller M, Cloughesy T, Perry JR, Wick W. Standards of care for treatment of recurrent glioblastoma - are we there yet? Neuro. Oncol. 15, 4-27 (2013).

8. Montemurro N, Perrini P, Blanco MO, Vannozzi R. Second surgery for recurrent glioblastoma: a concise overview of the current literature. Clin. Neurol. Neurosurg. 142, 60-64 (2016).

9. Greco WR, Bravo G, Parsons JC. The search for synergy: a critical review from a response surface perspective. Pharmacol. Rev. 47, 331-385 (1995).

10. Diaz RJ, Ali S, Qadir MG, De La Fuente MI, Ivan ME, Komotar RJ. The role of bevacizumab in the treatment of glioblastoma. J. Neurooncol. 133, 455-467 (2017).

11. Abrams DA, Hanson JA, Brown JM, Hsu FP, Delashaw JB, Bota DA Jr. Timing of surgery and bevacizumab therapy in neurosurgical patients with recurrent high grade glioma. J. Clin. Neurosci. 22, 35-39 (2015).

12. de Lemos ML, Markarian A, Chan E, Schaff K, Walisser S. Clinical effectiveness of bevacizumab in patients with recurrent brain tumours: a population-based evaluation. J. Oncol. Pharm. Pract. 24, 33 -36 (2016).

13. Wang Y, Xing D, Zhao M, Wang J, Yang Y. The role of a single angiogenesis inhibitor in the treatment of recurrent glioblastoma multiforme: a meta-analysis and systematic review. PLoS ONE 11, e0152170 (2016).

14. Hofman FM, Stathopoulos A, Kruse CA, Chen TC, Schijns VE. Immunotherapy of malignant gliomas using autologous and allogeneic tissue cells. Anticancer Agents Med. Chem. 10, 462-470 (2010).

15. Jackson CM, Lim M, Drake CG. Immunotherapy for brain cancer: recent progress and future promise. Clin. Cancer Res. 20, 3651-3659 (2014).

16. Stathopoulos A, Samuelson C, Milbouw G, Hermanne JP, Schijns VE, Chen TC. Therapeutic vaccination against malignant gliomas based on allorecognition and syngeneic tumor antigens: proof of principle in two strains of rat. Vaccine 26, 1764-1772 (2008).

17. Bota DA, Alexandru-Abrams D, Pretto C et al. Use of ERC-1671 vaccine in a patient with recurrent glioblastoma multiforme after progression during bevacizumab therapy: first published report. Perm. J. 19, 41-46 (2015).

18. Schijns VE, Pretto C, Devillers L et al. First clinical results of a personalized immunotherapeutic vaccine against recurrent, incompletely resected, treatment-resistant glioblastoma multiforme (GBM) tumors, based on combined allo- and auto-immune tumor reactivity. Vaccine 33, 2690-2696 (2015). 
19. Okada H, Weller M, Huang R et al. Immunotherapy response assessment in neuro-oncology: a report of the RANO working group. Lancet. Oncol. 16, e534-e542 (2015).

20. Wen PY, Macdonald DR, Reardon DA et al. Updated response assessment criteria for high-grade gliomas: response assessment in neuro-oncology working group. J. Clin. Oncol. 28, 1963-1972 (2010).

21. National Cancer Institute. Common Terminology Criteria for Adverse Events (CTCAE) version 4.0. National Institute of Health, U.S. Department of Health and Human Services. 2009.

22. Sistigu A, Viaud S, Chaput N, Bracci L, Proietti E, Zitvogel L. Immunomodulatory effects of cyclophosphamide and implementations for vaccine design. Semin. Immunopathol. 33, 369-383 (2011).

23. Daenen LG, Shaked Y, Man S et al. Low-dose metronomic cyclophosphamide combined with vascular disrupting therapy induces potent antitumor activity in preclinical human tumor xenograft models. Mol. Cancer Ther. 8, 2872-2881 (2009).

24. Chang DZ, Lomazow W, Joy Somberg C, Stan R, Perales MA. Granulocyte-macrophage colony stimulating factor: an adjuvant for cancer vaccines. Hematology 9, 207-215 (2004).

25. Min L, Mohammad Isa SA, Shuai W et al. Cutting edge: granulocyte-macrophage colony-stimulating factor is the major $\mathrm{CD} 8^{+} \mathrm{T}$ cell-derived licensing factor for dendritic cell activation. J. Immunol. 184, 4625-4629 (2010).

26. Gabrilovich DI, Chen HL, Girgis KR et al. Production of vascular endothelial growth factor by human tumors inhibits the functional maturation of dendritic cells. Nat. Med. 2, 1096-1103 (1996).

27. Osada $\mathrm{T}$, Chong G, Tansik $\mathrm{R}$ et al. The effect of anti-VEGF therapy on immature myeloid cell and dendritic cells in cancer patients. Cancer Immunol. Immunother. 57, 1115-1124 (2008).

28. Taal W, Oosterkamp HM, Walenkamp AME et al. Single-agent bevacizumab or lomustine versus a combination of bevacizumab plus lomustine in patients with recurrent glioblastoma (BELOB trial): a randomised controlled Phase II trial. Lancet Oncol. 15, 943-953 (2014).

29. Field KM, Simes J, Nowak AK et al. Randomized Phase II study of carboplatin and bevacizumab in recurrent glioblastoma. Neuro. Oncol. 17, 1504-1513 (2015).

30. Heiland DH, Masalha W, Franco P, Machein MR, Weyerbrock A. Progression-free and overall survival in patients with recurrent glioblastoma multiforme treated with last-line bevacizumab versus bevacizumab/lomustine. J. Neuro-Oncol. 126, 567-575 (2016).

31. Pardoll DM, Topalian SL. The role of CD4(+) T-cell responses in antitumor immunity. Curr. Opin. Immunol. 10, 588-594 (1998).

32. Pulaski BA, Mcadam AJ, Hutter EK, Biggar S, Lord EM, Frelinger JG. IL-3 enhances development of tumor-reactive cytotoxic-cells by a CD4-dependent mechanism. Cancer Res. 53, 2112-2117 (1993).

33. McKinstry KK, Strutt TM, Swain SL. The potential of CD4 T-cell memory. Immunology 130, 1-9 (2010).

34. Crane CA, Han SJ, Ahn B et al. Individual patient-specific immunity against high-grade glioma after vaccination with autologous tumor derived peptides bound to the 96 KD chaperone protein. Clin. Cancer Res. 19, 205-214 (2013).

35. Ladomersky E, Zhai L, Lenzen A et al. IDO1 inhibition synergizes with radiation and PD-1 blockade to durably increase survival against advanced glioblastoma. Clin. Cancer Res. 24, 2559-2573 (2018).

36. Kong Z, Wang Y, Ma W. Vaccination in the immunotherapy of glioblastoma. Hum. Vaccin. Immunother. 14, 255-268 (2017).

37. Tivnan A, Heilinger T, Lavelle EC, Prehn JH. Advances in immunotherapy for the treatment of glioblastoma. J. Neurooncol. 131, 1-9 (2017).

38. Dang Y, Wagner WM, Gad E et al. Dendritic cell-activating vaccine adjuvants differ in the ability to elicit anti-tumor immunity due to an adjuvant specific induction of immune suppressive cells. Clin. Cancer Res. 18, 3122-3131 (2012). 
